\title{
Development and Fuzzy Sliding Mode Compensation Control of a Power Assist Lower Extremity Exoskeleton
}

\author{
Xinliang LU*, Fengpo DU**, Xingsong WANG***, Shan JIA****, Fengyu XU***** \\ *School of Mechanical Engineering, Shijiazhuang Tiedao University, Shijiazhuang 050043, China, E-mail:xinref@126.com \\ **School of Mechanical Engineering, Southeast University, Nanjing 211189, China, E-mail: 230109036@seu.edu.cn \\ ***School of Mechanical Engineering, Southeast University, Nanjing 211189, China, E-mail: xswang@seu.edu.cn \\ ****School of Mechanical Engineering, Southeast University, Nanjing 211189, China, E-mail: 230099043@seu.edu.cn \\ *****Nanjing University of Posts and Telecommunications, Nanjing 210023, China, E-mail: xufengyu598@163.com \\ cross $^{\text {ref }}$ http://dx.doi.org/10.5755/j01.mech.24.1.14564
}

\section{Introduction}

An exoskeleton is an electromechanical device which can be worn by users for power augmentation or rehabilitation and can walk in concert with the operator's locomotion [1]. It have extensive applications in civil and military field, including rehabilitation training for the patients, helping the elderly or the disabled one to walk without feeling any weakness, assisting the operator to lift more payloads (e.g. ammunition) in military field. Due to a broad prospect in application, it has attracted a lot of researchers' attention in the community of robotics around the world.

Berkeley lower extremity exoskeleton (BLEEX) which is used to augment human strength and endurance during locomotion was developed in University of California, Berkeley. BLEEX utilized hydraulic driving mode and linear hydraulic actuators were employed to drive each joint. The sensitivity amplification controller was employed to control BLEEX and this control method heavily depends on accurate dynamics of BLEEX, which is stable under specific operating conditions [2-4]. HAL (Hybrid Assistive Leg) was developed at University of Tsukuba in Japan. It can increase wearer's joint torque directly to lift heavy loads. It utilizes DC to drive each joint and uses a lot of sensors to acquire the motion intention of operator [5-6]. XOS2 which is developed by Raytheon is a whole body power assist exoskeleton and is considered as the most successful load-carrying exoskeleton around the world. Furthermore, exoskeletons which were reported publicly include HERCULE, HULC, Locomat [7-8].

PID is widely used in industrial control and robot control. In some cases, PID algorithm cannot achieve satisfactory results due to its bad adaptability to external environment [9]. So many researchers proposed different robust control algorithms to solve this problem, e.g. adaptive control, variable structure, fuzzy control. Another approach is to combine these advanced control algorithm and PID control algorithm. Then, some improved PID control algorithm emerged, for example, adaptive PID, fuzzy PID, or other intelligent PID control algorithms. Some exoskeletons adopted improved PID algorithm, e.g. the upper exoskeleton PERCRO L-Exos used slide mode PD controller [10-11].

The sliding mode control (SMC) was firstly proposed and elaborated by S.V. Emelyanov, then further developed by V. Utkin in the last century. Because of its robustness with respect to uncertainties and external disturb- ances, it has attracted a great attention of researchers who engaged in the study of control field. The engineering practices show that SMC is an efficient tool to cope with complex nonlinear dynamic plants operating under uncertainties [12-13]. For example, Song [14] applied the sliding mode control based on RBF neural network to a lower limb exoskeleton suit.

E.H. Mamdani successfully applied fuzzy logic which was proposed by Zadeh to the stream engine control system in 1974, which marked the emergence of fuzzy control. Thereafter, as an intelligent control method, it was further developed by other researchers, including Takagi and Sugeno who proposed the famous method of T-S fuzzy inference [15-17]. However, the fuzzy logic can be understood and implemented by computer conveniently, and other superiority over conventional method, including high robustness to system uncertainties and insensitiveness to imprecise dynamics of plant, that make it to be another effective method to nonlinear systems [18].

This paper presents a wearable exoskeleton for augmentation which is developed in the authors' university, including the configuration of mechanism and control system of exoskeleton. Furthermore, the configuration of control system is also presented in detail. In this paper, the sliding mode control algorithms with fuzzy compensation were presented and were employed in the exoskeleton joints control. The experimental results show that it is effective.

The remainder of this paper is organized as follows. The mechanical structure is presented in Section 2. The dynamics of the exoskeleton system is described in Section 3. The control architecture is introduced in Section 4. The controller design and stability verification based on the Lyapunov method are presented in Section 5. The experimental results of the exoskeleton tracking performance are showed and analyzed in Section 6. At the end of this paper, the conclusion is drawn in Section 7.

\section{Mechanical structure}

We developed a power assistant lower extremity exoskeleton (SPAEX) which is showed in Fig. 1, where the left one is the three-dimensional modeling diagram and the right one is the prototype. SPAEX is designed to have seven degrees of freedom (DOF) in the sagittal plane, including hip joints, knee joints and ankle joints. However, there is a small range of passive movement at the hip joints in transverse plane. The hip joints are actuated by double-tendon-sheath and the knee joints are driven by hydraulic actuators directly. In order to adapt to different one, 
the length of thigh and shank can be adjusted according to the operator. A spring is mounted at the ankle to store some energy at the negative work stage while walking.

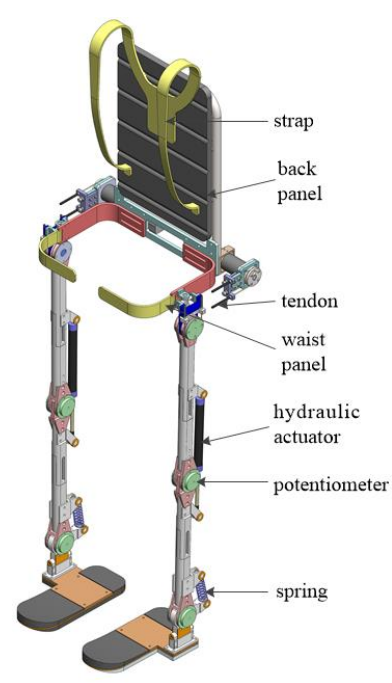

a

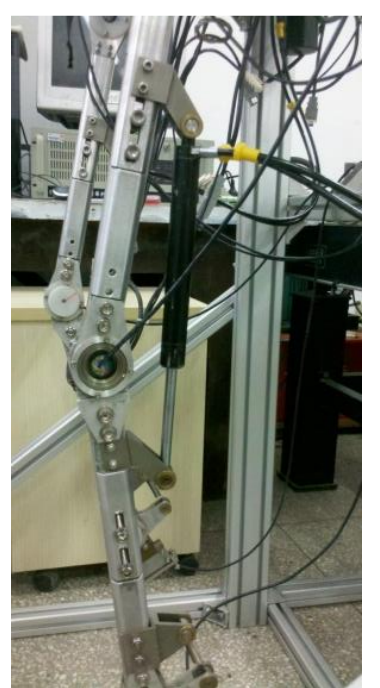

b

\section{Dynamics of exoskeleton}

Previously, the walking states of exoskeleton were divided into three stages (including BLEEX) around the world: single support, double support and double support with one redundancy [3].

However, the latter stages can be combined to one stage for the convenience of modeling, namely double support stage. Then, the walking states of exoskeleton are divided into two phases as shown in Fig. 2.

Single support: one leg is in stance phase and another is in swing phase.

SPAEX is modeled as a seven DOF serial link mechanism in the sagittal plane.

Double support: both legs are in stance phase.

The SPAEX is modeled as two planar four DOF serial link mechanisms that are connected to each other in the torso section.

The dynamics of exoskeleton is formulated in these two distinct walking states with the classical Lagrangian method and was presented in authors' previous paper [19].

Fig. 1 Exoskeleton(SPAEX):a - exoskeleton model; b - prototype of exoskeleton

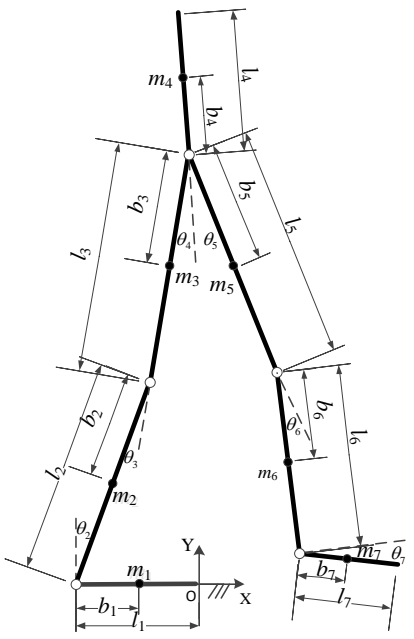

a

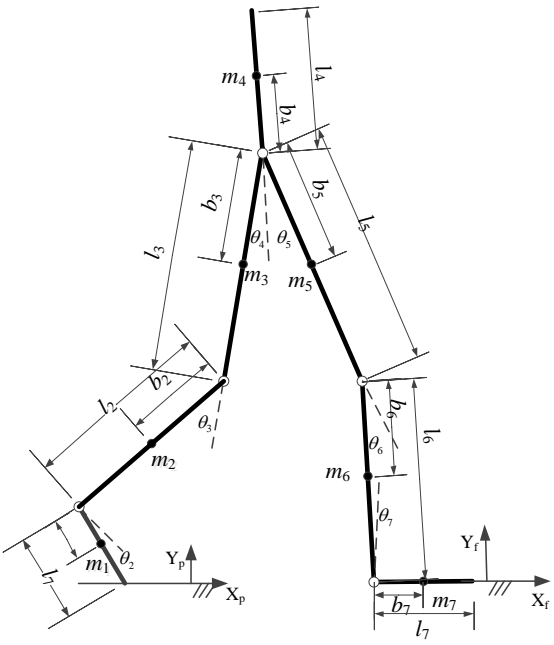

b

Fig. 2 The dynamic model of lower extremity exoskeleton: a - single support; b - double support

Finally, the dynamics of SPAEX can be expressed in the canonical form:

$$
D(\theta) \ddot{\theta}+C(\theta, \dot{\theta})+G(\theta)=\tau
$$

where $\theta, \dot{\theta}, \ddot{\theta}$ are the joint angles, angular velocities, and accelerations of SPAEX, respectively. $D(\theta)$ is an inertia matrix. $D(\theta)$ is a symmetric positive definite matrix and is bounded, $C(\theta, \dot{\theta})$ is the centripetal and Coriolis matrix. $G(\theta)$ is the gravitational torque, $\tau$ is the external torque to drive the exoskeleton, $\theta=\left[\theta_{1}, \theta_{2}, \cdots, \theta_{7}\right]^{T}$.

There is a positive real constant $\alpha$, the following inequality is satisfied in any condition.

$$
0<D(\theta)<\alpha I
$$

Theorem 1. The inertia matrix $D(\theta)$, centripetal and Coriolis matrix $C(\theta, \dot{\theta})$ satisfy the following equation:

$$
\dot{\theta}^{T}(D-2 C) \dot{\theta}=0,
$$

\section{Control architecture}

The control architecture of SPAEX as shown in Fig. 3 is designed. The entire control system mainly includes decision controller, joints controller and sensor data acquisition which includes the foot force sensors, joint angle sensors, two force sensors between human and exoskeleton which is used to identify the motion intention of 
the operator. The decision controller module generates the desired joints trajectory on the basis of the force sensors between human and the exoskeleton. Joints controller generates control commands to drive each joint actuator based on the decision controller output and sensor data. From the hierarchy point of view, the decision controller is the highest level controller. The joints controller which directly controls the position of exoskeleton joints is the low level controller.

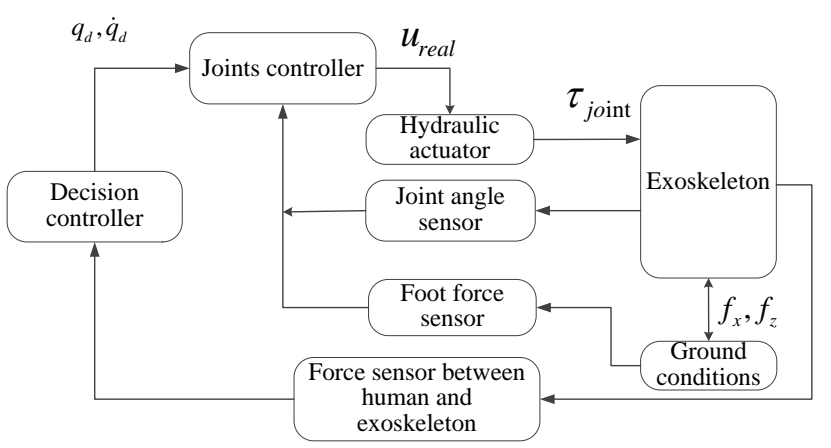

Fig. 3 Schematic of control architecture

The schematic of joints controller is shown in Fig. 4 and will be elaborated in Section 5.

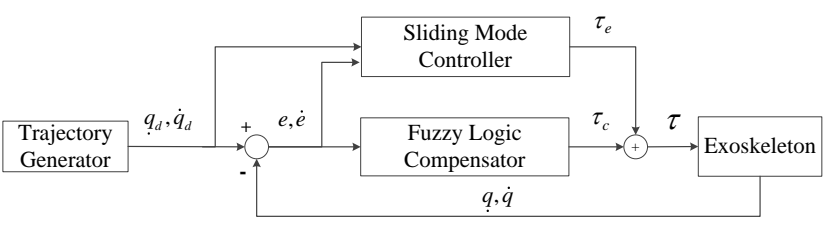

Fig. 4 Schematic of joints controllor

\section{Controller design}

In order to ensure the exoskeleton to follow the human while walking, the motion of each joint need to track the desired trajectory in real time. The exoskeleton joint controller is composed of the sliding mode controller and fuzzy logic compensator as shown in Fig. 3. The main controller is implemented by sliding mode control method and fuzzy logic compensator is employed to diminish the errors that are produced by uncertainties.

$x_{1}=\theta, x_{2}=\dot{\theta}$, are the state variables of joints, $x_{d}$ is the desired trajectory of joints which is determined by the interactive force between the exoskeleton and human, and $e=x_{1}-x_{d}$ is the tracking errors. The tracking objective is $e \rightarrow 0$ as $t \rightarrow \infty$.

The dynamics of exoskeleton can be written as canonical state equation:

$$
\left.\begin{array}{l}
\dot{x}_{1}=x_{2} ; \\
\dot{x}_{2}=D^{-1}\left(x_{1}\right) \tau-D^{-1}\left(x_{1}\right) C\left(x_{1}, x_{2}\right) x_{2}- \\
-D^{-1}\left(x_{1}\right) G\left(x_{1}\right)-D^{-1}\left(x_{1}\right) d(x) .
\end{array}\right\}
$$

The sliding mode variable structure method is chosen to design the exoskeleton controller because of its excellent anti-disturbance capability. Generally, the sliding mode controller consists of equivalent control and switch control $[13,20]$. However, a suitable sliding mode hyper- plane should be designed for the controller firstly, and the designed controller should guarantee the variables of state move towards and stay in the hyperplane.

[21]:

The sliding mode hyperplane is defined as follows

$$
s=\dot{e}+B e,
$$

where

$$
B=\left[\begin{array}{cc}
b_{1} & 0 \\
0 & b_{2}
\end{array}\right], b_{1}>0 \text { and } b_{2}>0 \text {, }
$$

Then, the derivative of $s$ is:

$$
\begin{aligned}
& \dot{s}=\ddot{e}+B \dot{e}=\ddot{x}_{1}-\ddot{x}_{d}+B\left(\dot{x}_{1}-\dot{x}_{d}\right)=B\left(\dot{x}_{1}-\dot{x}_{d}\right)- \\
& -\ddot{x}_{d}+D^{-1} \tau-D^{-1} C\left(x_{1}, x_{2}\right) x_{2}-D^{-1} G\left(x_{1}\right)-D^{-1} d(x),
\end{aligned}
$$

where the symbols $D\left(x_{1}\right), D^{-1}\left(x_{1}\right), G\left(x_{1}\right)$ are simplified as $D, D^{-1}, G$ and used in this paper below, respectively. In fact, the external disturbance $d(x)$ is unknown and variant, and error of the dynamics of the exoskeleton is also existed. The fuzzy logic systems have strong capabilities and can almost approximate any continuous function [22]. So the fuzzy logic system is taken into consideration to approximate the actual value of $d(x)$.

As shown in Fig. 5, a basic fuzzy logic system consists of a fuzzifier, some fuzzy IF-THEN rules base, a fuzzy inference engine and a defuzzifier, generally [23-26].

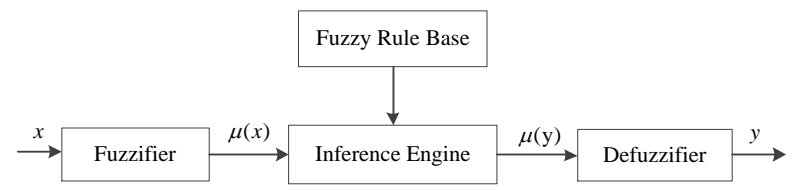

Fig. 5 Schematic of fuzzy logic system

The inputs of fuzzy logic compensator are $e$ and $\dot{e}$

The input and output variables membership functions (MFs) in fuzzy system are all chose to be the Gaussian functions.

$$
\mu_{A_{i}^{l}}\left(x_{i}\right)=\exp \left[-\left(\frac{x_{i}-z_{i}^{l}}{w_{i}^{l}}\right)^{2}\right],
$$

where, $A_{i}^{l}$ is the linguistic value, $z_{i}^{l}, w_{i}^{l}$ are the centers and widths of $l$-th Gaussian function in domains.

IF-THEN rules are expressed as follows:

$$
\begin{aligned}
& R_{i}: \text { IF } x_{1} \text { is } A_{1}^{i}, x_{2} \text { is } A_{2}^{i}, x_{3} \text { is } A_{3}^{i}, \cdots, x_{m} \text { is } A_{m}^{i} \\
& \text { THEN } y \text { is } B^{i}, i=1,2,3, \cdots, n .
\end{aligned}
$$

The detailed fuzzy tuning rules of compensator are indicated in Table 1. The tracking errors and the change rates of the errors are the inputs, the compensation torque are outputs, respectively. The symbols NL, NM, ZE, PM, and PL stand for negative large, negative medium, zero, 
positive medium, and positive large, respectively.

Table 1

The fuzzy rules of compensation control

\begin{tabular}{|c|c|c|c|c|c|c|}
\hline \multirow{2}{*}{\multicolumn{2}{c|}{$u$}} & \multicolumn{5}{c|}{$\dot{e}$} \\
\cline { 2 - 7 } & NL & NS & ZE & PS & PL \\
\hline \multirow{4}{*}{$e$} & NL & NL & NL & NS & NS & ZE \\
\cline { 2 - 7 } & NS & NL & NS & NS & ZE & PS \\
\cline { 2 - 7 } & ZE & NS & NS & ZE & PS & PS \\
\cline { 2 - 7 } & PS & NS & ZE & PS & PS & PL \\
\cline { 2 - 7 } & PL & ZE & PS & PS & PL & PL \\
\hline
\end{tabular}

By using Singleton fuzzification, product inference engine and the center of gravity (COG) defuzzification method, the output of fuzzy system can be expressed in the following equation:

$$
y_{i}=\frac{\sum_{k=1}^{\mathrm{n}} t_{k} \prod_{i=1}^{m} \mu_{A_{i}^{k}}\left(x_{i}\right)}{\sum_{k=1}^{\mathrm{n}} \prod_{i=1}^{m} \mu_{A_{i}^{k}}\left(x_{i}\right)},
$$

where $x=\left(x_{1}, x_{1}, \cdots, x_{m}\right)^{T}=\left(e, \dot{e}, \cdots, e^{(m)}\right)^{T} \in R^{m}$ is the input vector of fuzzy system, $y_{i}$ is the output vector of fuzzy system and the compensation torque of controller, $\mu_{A_{i}^{k}}\left(x_{i}\right)$ is membership function $A_{i}, t_{k}$ is a point of $B^{i}$ at which $y_{i} \in \mu_{B^{i}}(y)=1$.

$$
y_{i}=\theta^{\mathrm{T}} \xi(x)
$$

where: $\theta^{\mathrm{T}}=\left(t_{1}, t_{2}, \cdots, t_{n}\right)^{T}$ is the parameter vector.

$$
\begin{aligned}
& \xi(x)=\left(\xi_{1}(x), \xi_{2}(x), \cdots \xi_{m}(x)\right)^{T}, \\
& \xi_{k}(x)=\frac{\prod_{i=1}^{m} \mu_{A_{i}^{k}}\left(x_{i}\right)}{\sum_{k=1}^{\mathrm{n}} \prod_{i=1}^{m} \mu_{A_{i}^{k}}\left(x_{i}\right)} k=1,2, \cdots, m,
\end{aligned}
$$

The approximation errors are expressed as follows:

$$
e_{f}=d(x)-\hat{d}(x)
$$

And, the optimal approximation parameter is defined as follows:

$$
\Phi^{*}=\arg \min _{x \subset \Omega}\left\{\sup \left\|d(x)-\theta^{T} \xi(x)\right\|\right\} .
$$

The candidate Lyapunov function is defined as follows [22]:

$$
V(t)=\frac{1}{2}\left(s^{T} D s+\sum_{i=1}^{2} \tilde{\Phi}_{i}^{T} \alpha_{i} \tilde{\Phi}_{i}\right),
$$

where $\tilde{\Phi}_{i}=\Phi_{i}-\Phi_{i}^{*}$.
The derivative of $V(t)$ with respect to time is:

$$
\begin{aligned}
& \dot{V}(t)=s^{T} D \dot{s}+\frac{1}{2} s^{T} \dot{D} s+\sum_{i=1}^{2} \tilde{\Phi}_{i}^{T} a_{i} \dot{\tilde{\Phi}}_{i}= \\
& =s^{T}\left[D B\left(\dot{x}_{1}-\dot{x}_{d}\right)-D \ddot{x}_{d}+\tau-C\left(x_{1}, x_{2}\right) x_{2}-G-d(x)\right]+ \\
& +\frac{1}{2} s^{T} \dot{D} s+\sum_{i=1}^{2} \tilde{\Phi}_{i}^{T} a_{i} \dot{\tilde{\Phi}}_{i} .
\end{aligned}
$$

Substituting the Eq. (3) into (16), then:

$$
\begin{aligned}
& \dot{V}(t)=s^{T}\left[\begin{array}{l}
D B\left(\dot{x}_{1}-\dot{x}_{d}\right)-D \ddot{x}_{d}+\tau- \\
-C\left(x_{1}, x_{2}\right) x_{2}-G-d(x)
\end{array}\right]+ \\
& +C\left(x_{1}, x_{2}\right)+\sum_{i=1}^{2} \tilde{\Phi}_{i}^{T} \alpha_{i} \dot{\tilde{\Phi}}_{i} .
\end{aligned}
$$

The control laws are selected as follows:

$$
\begin{aligned}
& \tau=-D B\left(\dot{x}_{1}-\dot{x}_{d}\right)+D \ddot{x}_{d}-C\left(x_{1}, x_{2}\right) x_{2}+G+ \\
& +\hat{d}(x \mid \Phi)+C\left(x_{1}, x_{2}\right)\left(\dot{x}_{1}-\dot{x}_{d}\right)+C\left(x_{1}, x_{2}\right)\left(x_{1}-x_{d}\right)- \\
& -E \operatorname{sgn}(s)
\end{aligned}
$$

where $E$ is a matrix and $E=\operatorname{diag}\left(e_{1}, e_{2}\right), e_{1}>0, e_{2}>0$.

The adaption laws are:

$$
\dot{\Phi}_{i}=-\alpha_{i} s_{i} \xi(x), i=1,2,
$$

where; $\alpha_{i}$ are positive parameters.

Substituting the Eq. (18) into Eq. (17), then:

$$
\begin{aligned}
& \dot{V}(t)=s^{T}[d(x)-\hat{d}(x \mid \Phi)-E \operatorname{sgn}(s)]+\sum_{i=1}^{2} \tilde{\Phi}_{i}^{T} \alpha_{i} \dot{\tilde{\Phi}}_{i}= \\
& =s^{T}\left[e_{f}-E \operatorname{sgn}(s)\right]+\sum_{i=1}^{2}\left(\tilde{\Phi}_{i}^{T} \alpha_{i} \dot{\tilde{\Phi}}_{i}-s_{i} \tilde{\Phi}_{i}^{T} \xi(x)\right)= \\
& =s^{T}\left[e_{f}-E \operatorname{sgn}(s)\right]<0 .
\end{aligned}
$$

The approximation error of fuzzy system can be infinitely small, that is, $e_{f}$ can converge to neighborhood of zero. Then, the following inequality can be obtained:

$$
\dot{V}(t)<0 \text {. }
$$

$\dot{V}(t)$ is negative definite that guarantee the system is globally uniformly asymptotically stable.

In order to alleviate the adverse effect of chattering phenomenon of sliding mode control, a saturation function is used in place of the sign function $\operatorname{sgn}(s)$ in control laws. The saturation function is defined as follows:

$$
\operatorname{sat}\left(\frac{s}{\Delta}\right)=\left\{\begin{array}{l}
1, s>\Delta \\
\frac{s}{\Delta},|\Delta|<\Delta, \\
-1, s<\Delta
\end{array}\right.
$$

where $\Delta$ is the width of boundary layer of the sliding mode surface. However, the boundary layer $\Delta$ influences the 
control precision and robustness, so the size is determined specifically in the actual experiment.

\section{Experiment}

\subsection{Experiment preparation}

When $w_{i}^{l}=0.56$ and $z_{i}^{l}$ in Gaussian functions are chose as follows:

$$
z_{i}^{l}=-2,-1,0,1,2, \quad i=1,2,3,4,5 .
$$
in Fig. 6.

The Gaussian membership functions are illustrated

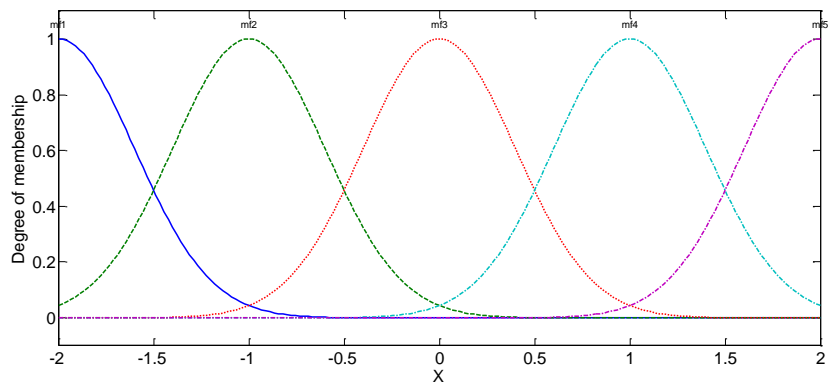

Fig. 6 Gaussian membership functions of the fuzzy compensation controller

Table 2.

The part of exoskeleton parameters are listed in

Table 2

The parameters of exoskeleton

\begin{tabular}{|c|c|c|}
\hline Symbols & Parameter meaning & Value \\
\hline$m_{2}=m_{6}$ & mass of the shank & $2.9 \mathrm{Kg}$ \\
\hline$m_{3}=m_{5}$ & mass of the thigh & $3.98 \mathrm{Kg}$ \\
\hline$m_{4}$ & mass of the torso & $4.19 \mathrm{Kg}$ \\
\hline$l_{2}=l_{6}$ & length of the shank & $386 \mathrm{~mm}$ \\
\hline$l_{3}=l_{5}$ & length of the thigh & $485 \mathrm{~mm}$ \\
\hline$l_{4}$ & length of the torso & $255 \mathrm{~mm}$ \\
\hline$b_{2}=b_{6}$ & mass centre of the shank & $188 \mathrm{~mm}$ \\
\hline$b_{3}=b_{5}$ & mass centre of the thigh & $236 \mathrm{~mm}$ \\
\hline$b_{4}$ & mass centre of the torso & $127 \mathrm{~mm}$ \\
\hline
\end{tabular}

\subsection{Experimental hardware}

As shown in Fig. 7, the experiment platform consists of two industrial computers and the exoskeleton mainly. The hardware architecture of the control system is presented in Fig. 8. The control hardware mainly is composed of two industrial personal computers(IPC-610L, Advantech Inc.), an analog input/output module (PCL-812PG, Advantech Inc.) which acquires data of sensors and output control signals, servo amplfiers, electro-hydraulic servovalves, hydraulic cylinders which are specifically designed for the exoskeleton, potentiometers which are mounted at the joints of exoskeleton for measuring the rotation angles. Two industrial personal computers communicate via The Ethernet or serial port and work in the mode of Matlab/Simulink Real-time Workshop. One computer is the host computer which is used to implement the control algorithm easily and the another computer is the target computer which is utilized to output the control commands in real-time.

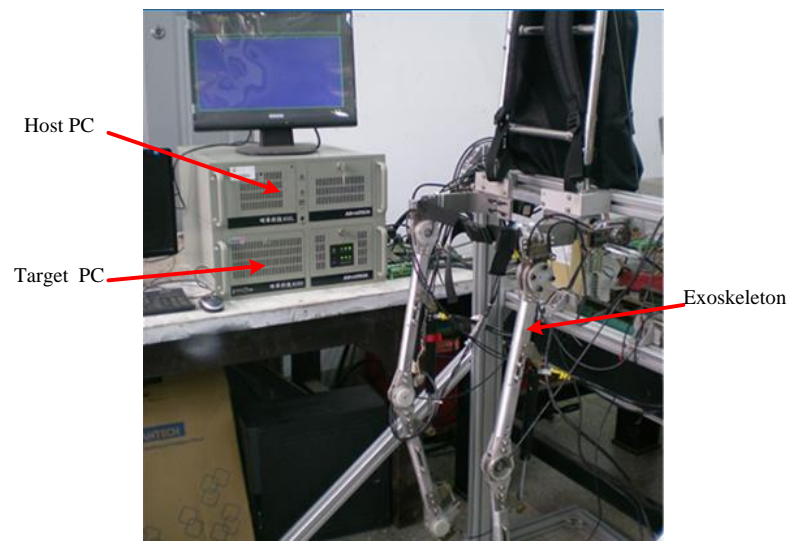

Fig. 7 Experiment platform

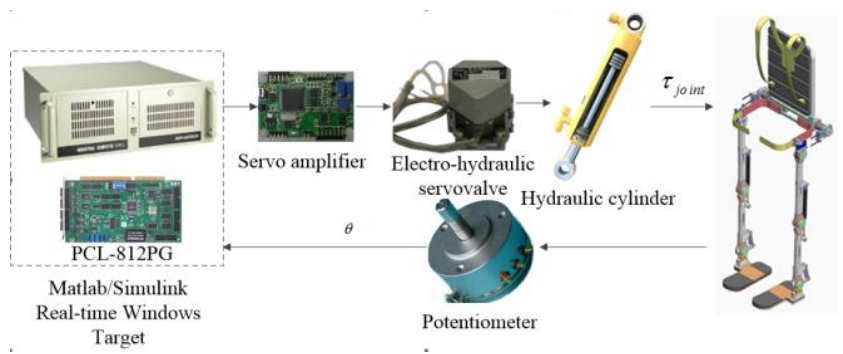

Fig. 8 The hardware architecture of the control system

The proposed controller is implemented in MATLAB/Simulink and Fig. 9 shows a simplified MATLAB controller scheme. A Bessel low pass analog filter with a cut-off frequency of $55 \mathrm{rad} / \mathrm{s}$ is used in MATLAB to attenuate the noises of velocity signal. The output of controller is restricted by a saturation module to produce voltage between $0-5 \mathrm{~V}$ because maximum input voltage of servo amplifier is $5 \mathrm{~V}$.

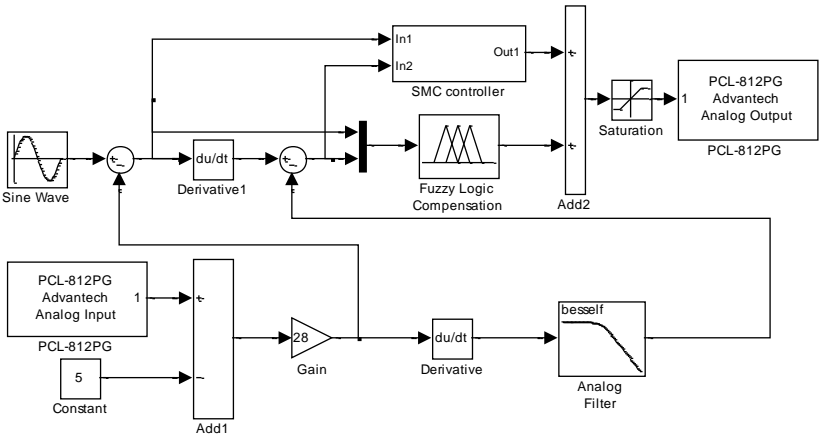

Fig. 9 The simplified MATLAB controller scheme

\subsection{Experiment results}

To verify the effectiveness of the proposed fuzzy compensation control scheme, some comparative study between the proposed FCSMC and the conventional PID method were completed in the experiments with different operating conditions, including with variable external loads and without external loads.

In the tracking experiments of exoskeleton hip joint and knee joint, the desired trajectory (DT) are set as 
sine curve $y=0.698 \sin (\pi t / 4)$, and $y=|0.8728 \sin (\pi t / 4)|$, respectively. Both of well-tuned PID and the proposed algorithm can get good tracking results without external loads in hip and knee experiments as shown in Fig. 10 and Fig. 14.

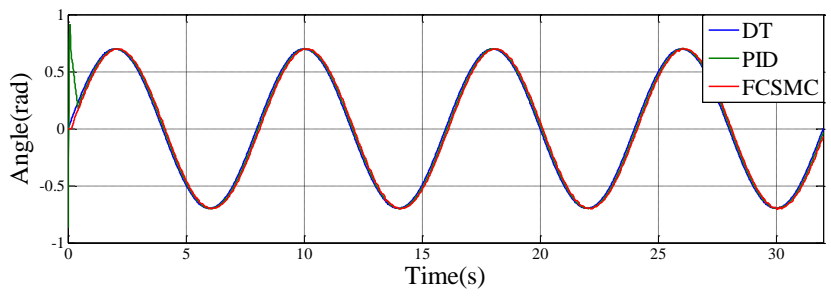

Fig. 10 The hip joint tracking without external loads

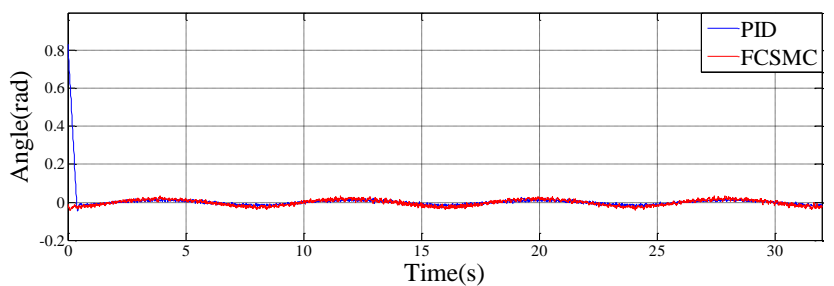

Fig. 11 The tracking errors of hip joint without external loads

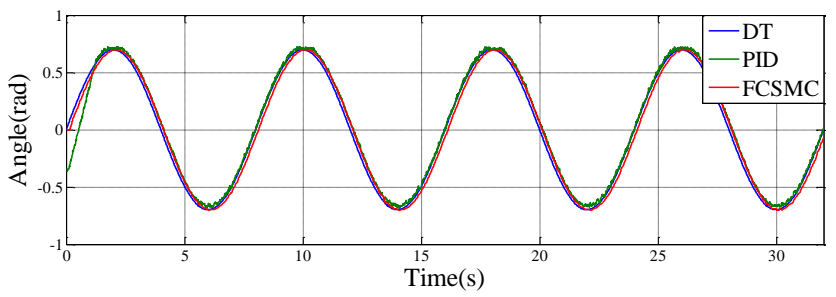

Fig.12 The hip joint tracking with variable external loads

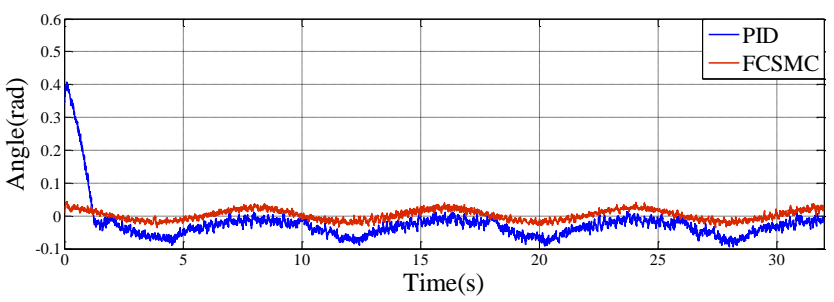

Fig. 13 The tracking errors of hip joint with variable external loads

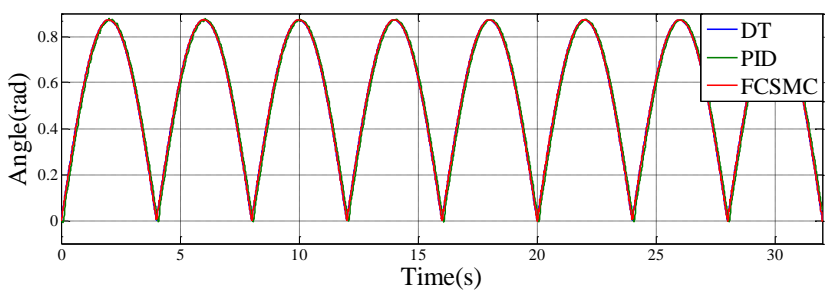

Fig. 14 The knee joint tracking without loads

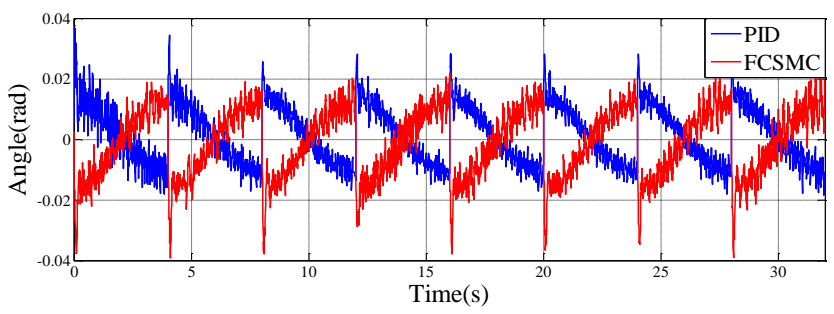

Fig. 15 The tracking errors of knee joint without loads

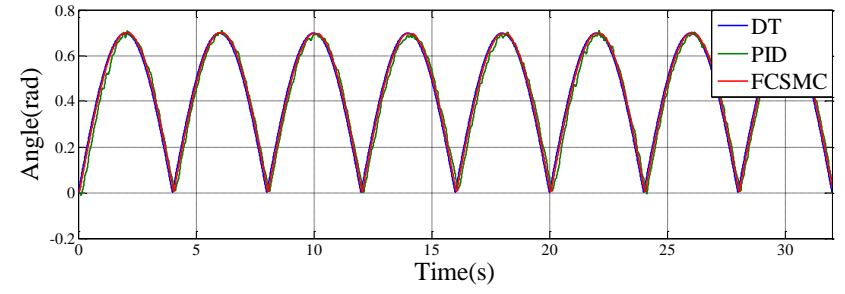

Fig. 16 The knee joint tracking with variable loads

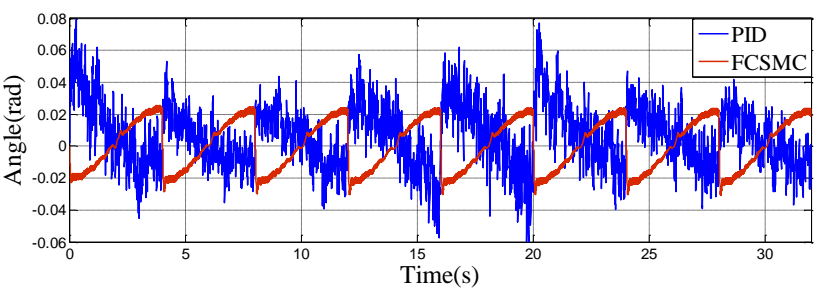

Fig. 17 The errors of knee joint with variable loads

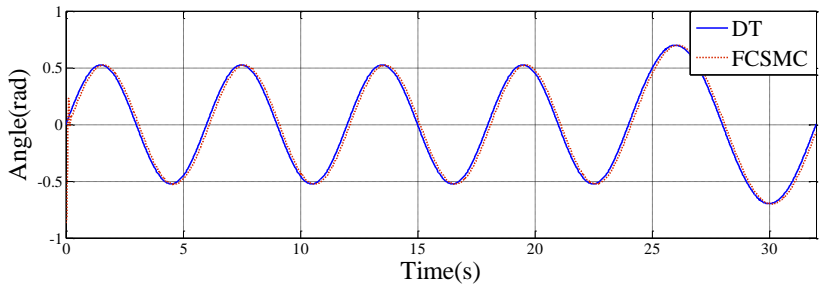

Fig. 18 The tracking results with FCSMC scheme at the variable frequency and amplitude

Fig. 11 and Fig. 15 show the comparison of errors. However, FCSMC can obtain better tracking results and have relatively small errors compared with PID with variable external loads as shown in Fig. 12 and Fig. 16. From Fig. 13 and Fig. 17, we can clearly see that the tracking errors with FCSMC are smaller than the errors with PID.

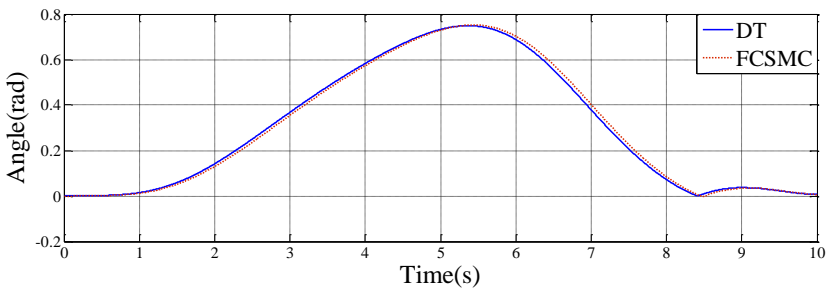

Fig. 19 The human gait tracking results of exoskeleton hip joint with FCSMC

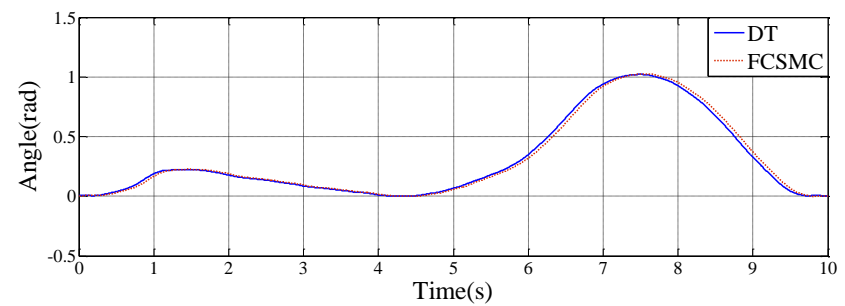

Fig. 20 The human gait tracking results of exoskeleton knee joint with FCSMC

Furthermore, some tracking experiments were completed with other trajectories to verify the proposed control scheme fully, including sine curve with variable frequency and amplitude and actual human gait trajectory. As shown in Fig. 18, the reference trajectories are $y=0.5236 \sin (\pi t / 3)$ before $24 \mathrm{~s}$ and $y=0.698 \sin (\pi t / 4)$ 
subsequently. As shown in Fig. 19 and Fig. 20, actual human gait hip and knee trajectories in accordance with the kinematic CGA data were also selected as the reference trajectory. Similarly, the tracking results are satisfactory and have acceptable errors.

From above experiments, the results indicate that the control performance and robustness of the proposed joints controller based on fuzzy compensation sliding mode control at variable conditions are better than that of the controller based on PID.

\section{Conclusion}

A power assist lower extremity exoskeleton (SPAEX) was developed in Southeast University, China. The mechanical design and architecture of control system were introduced in this paper. However, the conventional control method cannot be competent for the plants with inaccurate dynamics and uncertain external disturbances, e.g. PID control. The sliding mode control with fuzzy compensation algorithm of the exoskeleton was proposed to deal with this issue. According to the comparative experimental results, the proposed control scheme is effective and advantageous under the uncertain external disturbances. So we can conclude that the proposed control scheme can guarantee the requirements for high accuracy of the position tracking and be applied to the exoskeleton successfully.

As future work, we will try to coordinate the joints so that the exoskeleton can walk in concert with the operator.

\section{Acknowledgements}

This work is supported by National Nature Science Foundation of China (Grant No. 51575100, No. 51775284).

\section{References}

1. Dollar, A.M.; Herr, H. 2008. Lower extremity exoskeletons and active orthoses: challenges and state-of-the-art, IEEE Transactions on Robotics 24(1): 144-158. http://dx.doi.org/10.1109/TRO.2008.915453.

2. Zoss, A.B.; Kazerooni, H.; Chu, A. 2006. Biomechanical design of the Berkeley lower extremity exoskeleton (BLEEX), IEEE/ASME Transactions on Mechatronics 11(2): 128-138. http://dx.doi.org/10.1109/TMECH.2006.871087.

3. Kazerooni, H.; Racine, J.L.; Lihua, H.; Steger, R. 2005. On the control of the berkeley lower extremity exoskeleton(BLEEX), Proceedings of the 2005 IEEE International Conference on Robotics and Automation, p. $4353-4360$. http://dx.doi.org/10.1109/ROBOT.2005.1570790.

4. Kazerooni, H.; Steger, R.; Huang, L. 2006. Hybrid control of the berkeley lower extremity exoskeleton(BLEEX), The International Journal of Robotics Research 25(5): 561-573. http://dx.doi.org/10.1177/0278364906065505.

5. Kawamoto, H.; Sankai, Y. 2002. Comfortable power assist control method for walking aid by HAL-3. IEEE International Conference on Systems, Man and Cybernetics, $4 \mathrm{p}$.
http://dx.doi.org/10.1109/ICSMC.2002.1173328.

6. Kawamoto, H.; Suwoong, L.; Kanbe, S.; Sankai, Y. 2003. Power assist method for HAL-3 using EMG-based feedback controller, IEEE International Conference on Systems, Man and Cybernetics, 1648-1653.

http://dx.doi.org/10.1109/ICSMC.2003.1244649.

7. Renquan, L.; Zhijun, L.; Chun-Yi, S.; Anke, X. 2014. Development and learning control of a human limb with a rehabilitation exoskeleton, IEEE Transactions on Industrial Electronics 61(7): 3776-3785. http://dx.doi.org/10.1109/TIE.2013.2275903.

8. Onen, U.; Botsali, F.M.; Kalyoncu, M.; Tinkir, M.; Yilmaz, N. 2014. Design and actuator selection of a lower extremity exoskeleton, IEEE/ASME Transactions on Mechatronics 19(2): 623-632.

http://dx.doi.org/10.1109/TMECH.2013.2250295.

9. Pan, J.; Shi, G.; Zhu, X. 2010. Force tracking control for an electro-hydraulic actuator based on an intelligent feed forward compensator, Proceedings of the Institution of Mechanical Engineers, Part C: Journal of Mechanical Engineering Science 224(4): 837-849. http://dx.doi.org/10.1243/09544062JMES1795.

10. Frisoli, A.; Sotgiu, E.; Procopio, C.; Bergamasco, M. 2011. Design and implementation of a training strategy in chronic stroke with an arm robotic exoskeleton, IEEE International Conference on Rehabilitation Robotics (ICORR), 1-8. http://dx.doi.org/10.1109/icorr.2011.5975512.

11. Anam, K.; Al-Jumaily, A. 2012. Active Exoskeleton Control Systems: State of the Art, Procedia Engineering 41(1): 988-994. http://dx.doi.org/10.1016/j.proeng.2012.07.273.

12. Van, M.; Kang, H.; Shin, K. 2014. Backstepping quasi-continuous high-order sliding mode control for a Takagi-Sugeno fuzzy system with an application for a two-link robot control, Proceedings of the Institution of Mechanical Engineers, Part C: Journal of Mechanical Engineering Science 228(9): 1488-1500. http://dx.doi.org/10.1177/0954406213508936.

13. Utkin, V.; Guldner, J.; Shi, J. 2009. Sliding mode control in electro-mechanical systems. Taylor \& Francis Group.

https://doi.org/10.1201/9781420065619.

14. Song, S.; Zhang, X.; Tan, Z. 2014. RBF neural network based sliding mode control of a lower limb exoskeleton suit, Strojniski Vestnik-Journal of Mechanical Engineering 60(6): 437-446. http://dx.doi.org/10.5545/sv-jme.2013.1366.

15. LA, Z. 1965. Fuzzy sets, Information and control 8(3): 338-353. https://doi.org/10.1016/S0019-9958(65)90241-X.

16. Mamdani, E. 1974. Application of fuzzy algorithms for control of simple dynamic plant, Proceedings of the Institution of Electrical Engineers 121(12): 1585-1588. http://dx.doi.org/10.1049/piee.1974.0328.

17. Sugeno, M. 1985. Industrial Applications of Fuzzy Control, Elsevier Science Inc. 32p.

18. Kevin, M.; Stephen, Y. 1998. fuzzy control, California: Addison-Wesley Longman, Inc. 2p.

19. Jia, S.; Han, Y.; Lu, X.;Wang, X. 2014. Design of lower extremity exoskeleton based on analysis on special human gaits, Robot 36(4): 392-401. http://dx.doi.org/10.13973/j.cnki.robot.2014.0392. 
20. Liu, J. 2012. Sliding mode control design and matlab simulation, Beijing: Tsinghua University Press. 30p.

21. Mien, V.; Kang, H.; Shin, K. 2014. Adaptive fuzzy quasi-continuous high-order sliding mode controller for output feedback tracking control of robot manipulators, Proceedings of the Institution of Mechanical Engineers, Part C: Journal of Mechanical Engineering Science 228(1): 90-107. http://dx.doi.org/10.1177/0954406213490465.

22. Li, P.; Yang, G. 2009. Control of uncertain nonlinear systems against actuator faults using adaptive fuzzy approximation, American Control Conference, 4446-4451. http://dx.doi.org/10.1109/ACC.2009.5160349.

23. Purwar, S.; Kar, I.; Jha, A. 2004. Adaptive control of robot manipulators using fuzzy logic systems under actuator constraints, Proceedings of 2004 IEEE International Conference on Fuzzy Systems, 1449-1454. http://dx.doi.org/10.1109/fuzzy.2004.1375387.

24. Li, P.; Yang, G. 2010. A novel adaptive control approach for nonlinear strict-feedback systems using nonlinearly parameterised fuzzy approximators, International Journal of Systems Science 42(3): 517-527. http://dx.doi.org/10.1080/00207721003624576.

25. Euntai, K. 2004. Output feedback tracking control of robot manipulators with model uncertainty via adaptive fuzzy logic, IEEE Transactions on Fuzzy Systems 12(3): 368-378. http://dx.doi.org/10.1109/TFUZZ.2004.825062.

26. Byung Y.; Woon H. 2000. Adaptive control of robot manipulator using fuzzy compensator, IEEE Transactions on Fuzzy Systems 8(2): 186-199. http://dx.doi.org/10.1109/91.842152.
Xinliang Lu, Fengpo Du, Xingsong Wang, Shan Jia, Fengyu $\mathrm{Xu}$

\section{DEVELOPMENT AND FUZZY SLIDING MODE COMPENSATION CONTROL OF A POWER ASSIST LOWER EXTREMITY EXOSKELETON}

\section{S u m m a r y}

This paper presents a power assist lower extremity exoskeleton and a fuzzy sliding mode compensation controller. First, the mechanical design of this exoskeleton and the control configuration are introduced. Second, the dynamics of lower extremity exoskeleton is analyzed and the sliding mode controller with fuzzy compensation algorithm under uncertainties for exoskeleton joints is proposed. The theoretical proof shows that it is globally stable in the sense of Lyapunov. The experiments indicate that the proposed control scheme is effective; not only it ensures the control accuracy but also is robust to external uncertainties compared with PID control.

Keywords: exoskeleton, sliding mode, fuzzy control, compensation, robotics, trajectory tracking.

Received May 03, 2016 Accepted February 15, 2018 